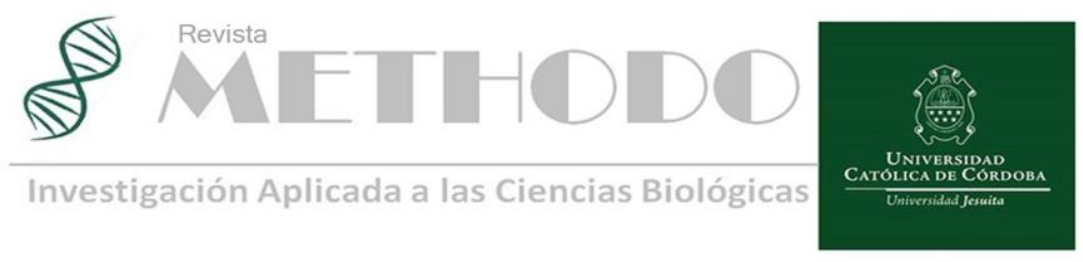

\title{
Tratamiento anticoagulante de la trombosis venosa profunda de miembro superior asociada a dispositivos intravasculares
}

\section{Deep vein thrombosis of the upper limbs associated with intravascular devices}

\author{
Sr. Editor.
}

Posterior a la lectura del artículo original "trombosis venosa profunda de miembros superiores asociada a dispositivos intravasculares serie de casos" publicado en la Revista Methodo el 30 de junio de 2019, nos parece oportuno poder ampliar algunos conceptos sobre el tratamiento anticoagulante de la trombosis venosa profunda de miembro superior (TVPMS) asociada a catéter.

La colocación de dispositivos intravasculares predispone a la formación de trombosis ya que influye sobre los componentes de la tríada de Virchow: estasis venosa, hipercoagulabilidad e injuria vascular. Los factores de riesgo para TVPMS asociado a dispositivos intravasculares se pueden agrupar en: relacionados al catéter (múltiples intentos de inserción, sitio de colocación y características del catéter como ser diametro, tipo, número de lúmenes y material de fabricación) o factores de riesgo asociados al paciente y terapia recibida (neoplasia, trauma o cirugía reciente, historia de tromboembolismo venoso, insuficiencia renal, edad avanzada, inmovilización, quimioterapia, radioterapia, eritropoyetina y nutrición parenteral).

En la actualidad hay escasos ensayos clínicos controlados y randomizados sobre el tratamiento de TVPMS asociada a catéter, y la mayoría de las recomendaciones surgen de estudios observacionales o extrapolaciones de ensayos realizados en pacientes con TVP de miembro inferior ${ }^{1}$.
El objetivo principal del tratamiento anticoagulante en pacientes con TVPMS asociada a catéter es reducir la sintomatología, prevenir la extensión de la trombosis y mantener la funcionalidad del dispositivo intravascular.

El tratamiento de elección es anticoagulación. Se sugiere la no remoción del catéter si el mismo es necesario, funciona correctamente y se encuentra bien ubicado sin infección concomitante. En caso de tener que ser removido se sugiere anticoagular con un mínimo de 7 días previos para disminuir el riesgo de complicaciones tromboembólicas relacionadas a la extracción del mismo. Kovacs et al, realizó un estudio prospectivo con 74 pacientes con TVPMS asociada a catéter y tratamiento anticoagulante, mostrando que la mayoría de los catéteres permanecen funcionales y sin evidencia de trombosis recurrente ${ }^{2}$.

En pacientes con cáncer y TVPMS asociada a catéter el tratamiento anticoagulante de elección inicial es la heparina de bajo peso molecular (HBPM) o la heparina no fraccionada, aunque no hay estudios comparativos con warfarina en esta población de pacientes. Si el catéter es removido la duración del tratamiento debe ser por un mínimo de 3 meses. Por el contrario, se sugiere mantener anticoagulación hasta que el dispositivo sea extraído ${ }^{1}$.

En pacientes sin cáncer con TVPMS asociada a catéter las opciones terapéuticas pueden ser HBPM, antagonistas de la vitamina $\mathrm{K}$ o anticoagulantes orales de acción directa (DOACs). La última actualización de las guías

Revista Methodo: Investigación Aplicada a las Ciencias Biológicas. Facultad de Medicina.

Universidad Católica de Córdoba. Jacinto Ríos 571 Bo Gral. Paz. X5004FXS. Córdoba.

Argentina. Tel.: (54) 3514517299 / Correo: methodo@ucc.edu.ar / Web: methodo.ucc.edu.ar|

CARTAS AL EDITOR Methodo 2019;4(3):101-102. 
ACCP 2016 recomiendan los nuevos anticoagulantes sobre los antagonistas de la vitamina $\mathrm{K}$ (grado 2B) y antagonistas de la vitamina $\mathrm{K}$ sobre HBPM (grado $2 \mathrm{C}$ )3. Aunque no hay estudios publicados del uso de DOACs en TVPMS se extrapola el beneficio que tienen en estudios de TVP de miembro inferior con o sin tromboembolismo pulmonar ${ }^{3}$.

En referencia a la trombolisis, pacientes con cáncer tienen mayor riesgo de sangrado, por lo tanto, son excluidos de la mayoría de los estudios con trombolíticos. La utilización de trombolíticos se reserva para situaciones de trombosis masiva y trombosis refractaria a la anticoagulación. La utilización de Alteplase en bajas dosis podría utilizarse en catéteres no funcionales ${ }^{4}$.

\section{Gisela Máspero}

Soledad Molnarl

1Médica Hematóloga. Servicio de Hematología. Clínica Universitaria Reina Fabiola. Universidad Católica de Córdoba.

\section{Bibliografía}

1.Anita Rajasekhar and Michael B. Streiff. How I treat central venous access device-related upper extremity deep vein thrombosis. Blood 2017 129: 2727-2736.

2. Kovacs MJ, Kahn SR, Rodger M, et al. A pilot study of central venous catheter survival in cancer patients using low-molecular-weight heparin (dalteparin) and warfarin without catheter removal for the treatment of upper extremity deep vein thrombosis (The Catheter Study). J Thromb Haemost. 2007;5(8):16501653.

3.Kearon C, Akl EA, Ornelas J, et al. Antithrombotic therapy for VTE disease: Chest guideline and expert panel report [published correction appears in Chest. 2016;150(4):988]. Chest. 2016;149(2):315-352.

4. Tebbi C, Constanzi J, Shulman R, et al. A phase III, open-label, single-arm study of tenectaplase for restoration of function in dysfunctional central venous catheters. J Vasc Interv Radiol. 2011;22(8):1117-1123.

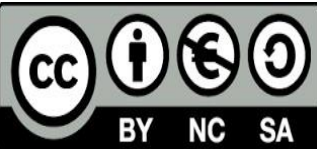

Revista Methodo: Investigación Aplicada a las Ciencias Biológicas. Facultad de Medicina. Universidad Católica de Córdoba. Jacinto Ríos 571 Bo Gral. Paz. X5004FXS. Córdoba. Argentina. Tel.: (54) 351 4517299 / Correo: methodo@ucc.edu.ar / Web: methodo.ucc.edu.ar| CARTAS AL EDITOR Methodo 2019;4(3):101-102 